\title{
Vorwort des Utbersetzers der 1. Auflage
}

Nachdem ich mich bereits an dem Vorläufer der hier vorliegenden "Analyse qualitative rapide des cations“, der "Méthode nouvelle d'analyse des cations" von G. Charlot und D. BÉzIER, Paris 1943, über die Durchführbarkeit der neuen Arbeitsweise orientiert, sie auch an einigen praktischen Beispielen erprobt hatte und nachdem ich gelegentlich feststellen konnte, daß in weiten Kreisen der beruflich tätigen Chemiker ein großes Interesse für eine schnelle Methode der qualitativen Analyse besteht, habe ich mich entschlossen, die Übersetzung dieses Werkchens des bekannten französischen Chemikers G. CharLot zu übernehmen. Ich erfreute mich dabei seiner und seiner Mitarbeiterin Mlle D. BÉzIER Unterstützung. Die nunmehr vorliegende deutsche Ausgabe stellt somit die neueste Fassung der Arbeitsweisen der beiden genannten Autoren sowie von R. GAUGUIN dar.

Anfang 1953 trat der Verlag Walter de Gruyter \& Co., Berlin, mit dem Vorschlag an mich heran, diese Ubersetzung zu einer „Qualitativen Schnellanalyse“ zu erweitern. Dazu bot sich mir die Arbeit eines meiner früheren Schüler und Mitarbeiter, J. M. Odekerken, s'Hertogenbosch, der selbst zwar nur indirekt Schüler von CHARLOT war, aber durch dessen oben genannte Werke angeregt, es versucht hatte, die Arbeitsweise auf die Ermittlung der Anionen auszudehnen. Dieser Versuch erschien als Auszug aus seiner Dissertation, Aachen 1950 in Zeitschrift für analytische Chemie „Über die Empfindlichkeit und Spezifität von Anionenreaktionen" Bd. 131 (1950) S. 165. 
Das Ziel der beiden Arbeiten ist das gleiche, nämlich die schnelle Orientierung über die vorliegenden Ionen beider Arten. Unter bewußtem Verzicht auf die Ermittlung kleinster Mengen derselben, also auf die sog. Spurensuche, mußte hier versucht werden, nicht nur die Empfindlichkeit, sondern auch die Spezifität der Reaktionen derart zu steigern, daß der Nachweis einzelner Ionenarten auch neben einer großen Menge aller anderer möglich ist. Die Arbeitsweise ist also eine mikroanalytische, aber sie verbindet damit den Vorteil, daß nicht große Substanzmengen, wie bei dem üblichen qualitativen Analysengang verarbeitet werden müssen und da $B$ somit alle langwierigen Filtrationen und Auswaschungen vermieden werden, wie auch die Verluste kleiner nicht erfaßbarer Mengen durch Einschließungen bei den Fällungen. Es wird also stets nur mit ganz wenigen Tropfen einer vorbereiteten Lösung gearbeitet, aber es muß darauf geachtet werden, daß Störungen, je nach Wahl des gewählten empfindlichen Reagenzes, in geeigneter Weise umgangen werden.

Der zeitliche Vorteil kommt vor allen Dingen der Kationenbestimmung zugute, da ja der Anionennachweis schon seit ehedem nicht in einem systematischen Gang, sondern hauptsächlich durch spezifische Reaktionen in Einzelproben erfolgt. Gleichwohl vereinfacht sich auch hier die Nachweisführung, besonders dann, wenn sie ohne vorherige Abtrennung der Kationen durchgeführt werden kann.

Die qualitative Schnellanalyse zerfällt somit in zwei Teile, deren erster den Kationen und deren zweiter den Anionen gewidmet ist. Ich habe mich bemüht, die Darstellung in beiden Teilen aufeinander abzustimmen; es wird jeweils die für die vorliegende Aufgabe als die beste gefundene Reaktion beschrieben, dann folgt die Angabe des Reagenzes, sodann die Ausführung der Reaktion und schließlich folgt die Beschreibung der Beseitigung der 
Störungen. - Bezüglich der von beiden Forschern durchgeführten Auswahl-Versuche verweise ich für den ersten Teil auf: Charlot, „Théorie et méthode nouvelle d'analyse qualitative“ Paris 1949 und für den zweiten auf die angeführte Dissertation.

Dem Verlag Walter de Gruyter danke ich für die Herausgabe des Ganzen in einer für den Gebrauch unmittelbar auf dem Laboratoriumstisch handlichen Form.

Aachen, im Sommer 1954

A. Schleicher

\section{Vorwort des Übersetzers der 2. Auflage}

Für die 2. Auflage war ich vor allen Dingen bemüht, die Bestimmung des Büchleins, so wie sie von Anfang an gedacht war, aufrechtzuerhalten; es soll eine Anweisung sein für den gelernten Analytiker und praktischen Chemiker, um sich auf schnellem Wege über die Zusammensetzung einer Probe an Metallen und Säureresten zu orientieren. Demgemäß konnte in ihr nicht allen Einwänden und Wünschen der Herren Kritiker der 1. Auflage nachgekommen werden. Gleichwohl aber ist die Bezeichnung dieser 2. Auflage als einer ,verbesserten und erweiterten" berechtigt. Dies kommt vor allen Dingen in dem Vorwort zur 2. Auflage der "Analyse qualitative rapide des cations" von G. CHarLor, D. Bezier und R. Gadgein, Paris 1954 zum Ausdruck. Hier heißt es: „Die Methode, so wie sie in der 1. Auflage beschrieben wurde, war insofern wenig befriedigend, als wir infolge Mangels an passenden Reaktionen gezwungen waren, zum Nachweis der Erdalkali- und Alkali-Metalle Trennungen vorzunehmen. Es mußte also die klassische Fällung aller übrigen Elemente 